\title{
Microchannel-patterned and heparin micro-contact-printed biodegradable composite membranes for tissue-engineering applications
}

\author{
Erkan T. Baran ${ }^{1,2 *}$, Kadriye Tuzlakoğlu ${ }^{1,2}$, António Salgado ${ }^{1,3}$ and Rui L. Reis ${ }^{1,2}$ \\ ${ }^{1}$ 3Bs Research Group - Biomaterials, Biodegradables and Biomimetics, University of Minho, Headquarters of the European Institute of \\ Excellence on Tissue Engineering and Regenerative Medicine, AvePark, S. Cláudio do Barco, 4806-909 Taipas, Guimarães, Portugal \\ ${ }^{2}$ Institute for Biotechnology and Bioengineering (IBB), PT Government Associated Laboratory, Guimarães, Portugal \\ ${ }^{3}$ Health and Life Sciences Research Institute, School of Health Sciences, University of Minho, Braga, Portugal
}

\begin{abstract}
Microchannel-patterned starch-poly(capro-lactone)/hydydroxyapatite (SPCL-HA) and starchpoly(lactic acid) (SPLA) composite membranes were produced for use as a laminated tissueengineering scaffold that incorporates both physical and biochemical patterns. For this purpose, SPCL (30\% starch) blended with inorganic hydroxyl apatite $(50 \%)$ and SPLA (50\% starch) membranes were made with compressive moulding. Consequently, the microchannel structures (width $102 \mu \mathrm{m}, 174 \mu \mathrm{m}$ intervals) were developed on the composite membranes by means of micro-patterned metal mould(s) and hydraulic pressing. An elastomer poly(dimetylsiloxane) stamp was used to transfer heparin as a biochemical cue over the microchannel surfaces by micro-contact printing $(\mu \mathrm{CP})$. Toluidine blue staining of developed capillaries and heparin $\mu \mathrm{CP}$-coated membranes showed that heparin was transferred predominantly over the microchannel surfaces. Fibroblast cell culture over the microchannel-formed and heparin $\mu$ CP-modified SPCL-HA and SPLA membranes showed distinct growth patterns. In contrast to the uniform cell layer formed on unmodified microchannels, the cells were bridging across the grooves of heparin-printed microchannels. At extended culture periods, the heparin-printed microchannels were covered with a layer of fibroblast cells without cellular ingrowths inside. This study indicated that the topographical pattern could induce an organization of fibroblasts only with the biochemical cue and the cells' functions can be controlled spatially over the microchannels by using both cues. Copyright $\odot 2010$ John Wiley \& Sons, Ltd.
\end{abstract}

Received 26 July 2010; Accepted 30 August 2010

Keywords microchannel; micropatterning; composite biomaterials; membranes; fibroblast; tissue engineering

\section{Introduction}

New and rapidly developing tissue-engineering technology requires novel processing methods and scaffold designs for better tissues in growth and rapid assimilation

\footnotetext{
*Correspondence to: Erkan T. Baran, 3Bs Research Group Biomaterials, Biodegradables and Biomimetics, University of Minho, Headquarters of the European Institute of Excellence on Tissue Engineering and Regenerative Medicine, AvePark, S. Cláudio do Barco, 4806-909 Taipas, Guimarães, Portugal. E-mail: turker.baran@dep.uminho.pt
}

inside implantation sites. Various scaffold-processing methodologies have been studied and fabricated, such as fibre bonding (Tuzlakoğlu et al., 2005; Santos et al., 2008), solvent casting (Silva et al., 2007), particulate leaching (Reignier and Huneault, 2006), freeze-drying (O'Brien et al., 2004) and melt moulding (Oh et al., 2003). With conventional preparation processes, however, the scaffolds can encounter problems, such as limitation to thin structures, residual particles and solvents in the polymer matrix, irregularly shaped pores and insufficient interconnectivity for cell infiltration and diffusion of nutrients (Zeltinger et al., 2001; Hollister et al., 
2002; Zmora et al., 2002). For those reasons, 3D scaffolds that are made by stacking multi-layered micro-patterned sheets or micro-patterned films and membranes featuring microchannels have recently proposed to solve the aforementioned diffusion-related problems (Papenburg et al., 2009; Cao et al., 2010).

Previously, Ostuni et al. (1999) studied the interaction of cells with chemically micro-patterned surfaces by micro-contact printing $(\mu \mathrm{CP})$ of self-assembled monolayers of alkenethiolates. This micro-patterning technique has been used on biomaterial surfaces to control cell adhesion spatially by printing aspartate-glycine-arginine (RGD) cell attachment peptide (Hasenbein et al., 2002), poly(ethylene glycol)-grafted polylysine (Csucs et al., 2003), laminin (Lauer et al., 2001) and non-adhesive/adhesive pluronic surfactant and extracellular matrix protein (Tan et al., 2004), respectively. In addition to chemical patterning, the topographical patterning of surfaces by means of several micro-fabrication techniques, such as photolithography and soft lithography, has been used for investigating and controlling cell functions (Kane et al., 1999; Mahoney et al., 2005; Flemming et al., 1999; Thakar et al., 2003).

A simultaneous effect of both chemical and physical micro-patterning on cell behaviour has been poorly investigated. In an earlier study, laminin, which was selectively adsorbed onto the grooves, was found to improve the adhesion of Schwann cells, while the microgrooves were found to align the cells along the direction of patterning (Miller et al., 2001). Barbucci et al. (2002) demonstrated that the two components of their micro-patterned system, viz. the surface chemistry (sulphated hyaluronic acid and glass) and topography (width and height of stripes), cannot tell definitely that which of these was the most important. Importantly, it was further highlighted by $\mu \mathrm{CP}$ micropatterning studies that the cell direction and cellular functions can be significantly influenced by the geometry, celladsorption pattern or shape of the cell that are critical for normal tissue morphogenesis and wound healing (Nobes et al., 1999; Adams and Schwartz, 2000; Berry et al., 2004; Lauffenburger and Horwitz, 1996; Parker et al., 2002; Manwaring et al., 2004; Ghibaudo et al., 2009).

In this study, a novel processing method, which is based on compressive moulding between micropatterned metal moulds at high pressure, was used to form grooves on particulate composite membranes with high inorganic hydroxyapatite contents. The conventional micro-patterning techniques, such as the hot embossing, soft lithography and solvent casting on a micro-patterned mould, cannot be applied to composite biomaterials that are made up of inorganic minerals, hydrophilic biopolymers and hydrophobic polyesters together. There is lack of information about the synergistic effect of chemical and topographical micro-patterning on cell organization when both cues are present in the same space. For this purpose, heparin as a biochemical cue was $\mu \mathrm{CP}$-printed on the grooves of composite membranes in order to evaluate the effect of both cues on the behaviour of fibroblast cells.

\section{Materials and methods}

\subsection{Membrane preparation and pressure-assisted micro-patterning}

A starch-poly(caprolactone) (SPCL; corn starch 30\% w/w; Novamont, Italy) powder was mixed with hydroxyapatite (HA; Plasma Biotal, UK) fine powder (average particle size $10.1 \mu \mathrm{m}, 50 \% \mathrm{w} / \mathrm{w})$. The blended powder was then homogenized thoroughly in a blender and placed into a rectangular mould $(7 \times 7 \mathrm{~cm})$ and pressure moulded, using a hydraulic press at $80^{\circ} \mathrm{C}$. The same technique was used for the preparation of starch-poly(lactic acid) (SPLA; corn starch $50 \% \mathrm{w} / \mathrm{w}$; Novamont, Italy) flat membranes from its powder at $140^{\circ} \mathrm{C}$. In order to prepare the mould with cylindrical grids, a stainless steel wire (diameter $102.4 \mu \mathrm{m}$ ) was glued onto preformed line patterns (with $174 \mu \mathrm{m}$ intervals) over a stainless metal plaque $(2.0 \times 1.2 \mathrm{~cm})$ using cyanoacrylate glue. For micro-patterning of SPCL-HA and SPLA, a membrane piece was placed between the micro-patterned mould(s) with either with one or two patterned moulds. Then pressure was applied using a hydraulic press for a certain time period (Figure 1A). Micro-patterning experiments were performed at room temperature and $50^{\circ} \mathrm{C}$ for SPCL and SPLA, respectively. In order to find optimal conditions for micro-patterning, the pressure and processing time were changed and optimized. The surface of the processed membranes was analysed by scanning electron microscopy (SEM).

\subsection{Micro-contact printing of heparin}

An elastomer stamp was obtained by careful placement of a self-curable poly(dimethyl siloxane) (PDMS) layer

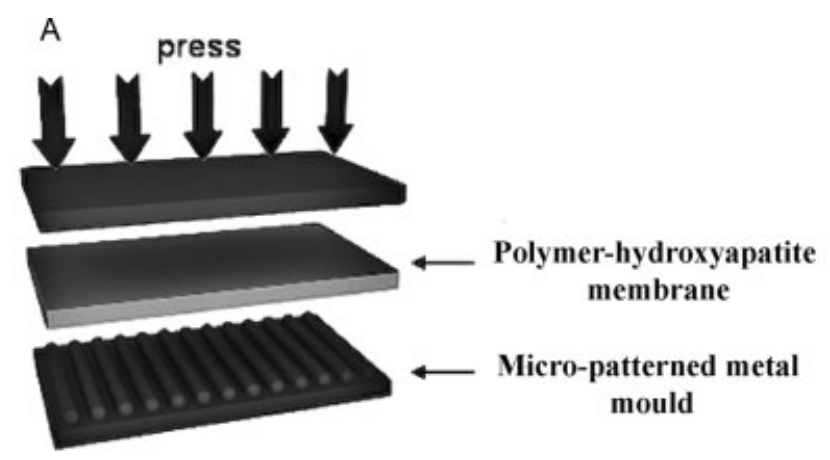

B

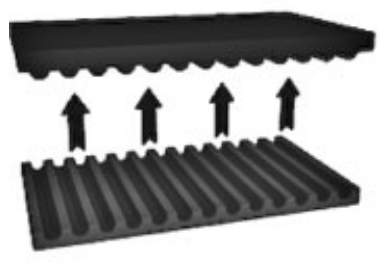
$\longleftarrow$ Heparin impregnated micro-patterned silicone mould

$\longleftarrow$ Micro-patterned and heparin printed polymerhydroxyapatite membrane

Figure 1. Micro-pattern formation (a) and consecutive $\mu \mathrm{CP}$ printing scheme (b) 

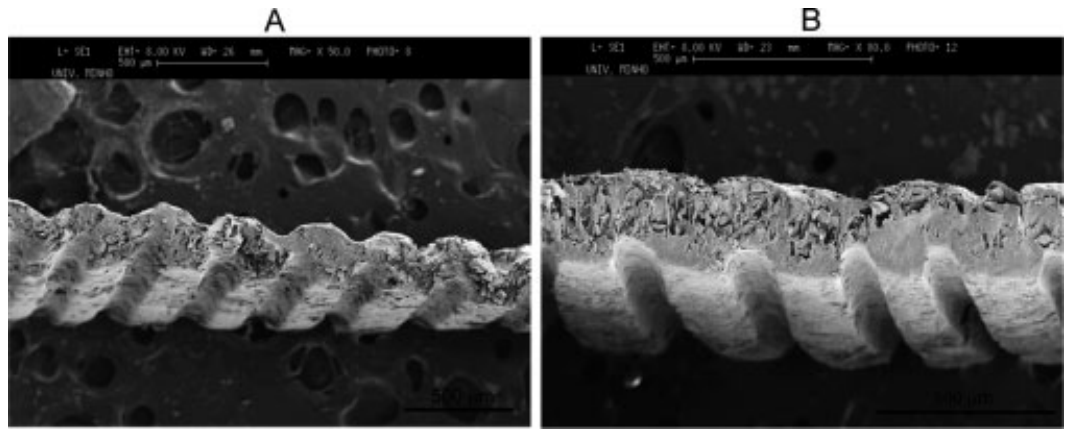

Figure 2. SEM photomicrographs of both sides patterned (a) and one side patterned (b) SPCL-HA membranes

over a replica of a metal mould. The obtained poly(dimethyl siloxane) stamp was used for $\mu \mathrm{CP}$ printing of the microchannel-patterned membranes. Since the hydrophobic nature of the silicone elastomer does not allow the wetting of the surface with heparin solution, 15 min of oxygen plasma treatment by a plasma reactor (Gala Instrumente, Germany) was carried out to make the stamp surface hydrophilic. The stamp was impregnated with heparin (porcine intestinal mucosa, Grade IA, from Sigma Chemical Co., St. Louis, MO, USA) solution ( $20 \mathrm{mg} / \mathrm{ml}$, with $0.5 \%$ chitosan solution). The chitosan (Sigma) was used to prevent the heparin being washed away in the tissue culture medium because of the adhesive effect of this hydrogel on solid surfaces and ionic complex formation with heparin. The stamp was then pressed gently over the micro-patterned membranes so that the ridges of the stamp could match and enter the grooves of the membrane (Figure 1B). After a few seconds of gentle manual pressing, the membrane was carefully removed from the stamp and allowed to dry at room temperature. For observation of heparin prints, the dried sample was immersed into an aqueous toluidine blue (Sigma) solution for about 5 min. After washing and drying, the stained samples were visualized under a stereo light microscope (Nikon SMZ-10).

\subsection{Cell culture experiments}

In order to observe the cell response on micropatterned membranes, a mouse lung fibroblasts cell line (L929) was selected. Before seeding on the membranes, the cells were trypsinized and resuspended in culture medium [Dulbecco's modified Eagle's medium (DMEM) high glucose, supplemented with $10 \%$ fetal bovine serum (FBS), 1\% antibiotics/antimicotics]. The cells were then seeded onto the membranes, using a density of $8 \times 10^{4}$ cells/membrane piece $\left(1 \mathrm{~cm}^{2}\right)$ and incubated in a humidified atmosphere at $37^{\circ} \mathrm{C}$ and $5 \% \mathrm{CO}_{2}$ for 2,3 and 7 days. The medium was renewed every 2 days. After each incubation period, membrane-cell constructs were fixed with glutaraldehyde and dehydrated in a graded series of ethanols for SEM analysis.

\subsection{SEM study}

The surfaces of the dried membrane samples, with or without cells cultured and fixed, were coated with an ultra-thin layer of gold under vacuum. The surface morphology of the samples was analysed by SEM (Leica Cambridge S360 microscope).

\section{Results and discussions}

\subsection{Fabrication of membranes with topographical micro-patterning}

In this study, a novel micro-fabrication method was developed for composite biomaterials which were composed of biodegradable polyesters, hydroxyapatite and starch. In order to process the membrane biomaterials, a micro-moulding process between micro-machined metal moulds under high pressure was used. The minimum requirements for micro-patterning were determined to be $1.25 \times 10^{3} \mathrm{~kg} / \mathrm{cm}^{2}$ and $1 \mathrm{~min}$ processing time for both SPCL-HA and SPLA membranes. At higher pressures the metal mould was observed to be deteriorated and corrupted patterns were obtained on the membranes. At lower pressures, however, unfinished and uneven pattern formations were detected.

From the SEM micrographs it was evident that microchannels were formed successfully with an average groove and ridge size of 102.4 and $174 \mu \mathrm{m}$, respectively (Figure 2A). SEM pictures showed embedded microchannel formations with sharp contours and the average depth of channels on SPCL-HA membranes was about $74 \mu \mathrm{m}$ (Figure 2A). In addition, it was possible to micro-pattern both sides of the SPCL-HA membranes by processing them between two micro-patterned metal moulds (Figure 2B).

\subsection{Surface characterization of heparin micro-contact-printed surfaces}

After micro-pattern formation, the microchannels of the membrane were spatially modified with heparin, using the $\mu \mathrm{CP}$ technique. Ridge structures of this elastic stamp 


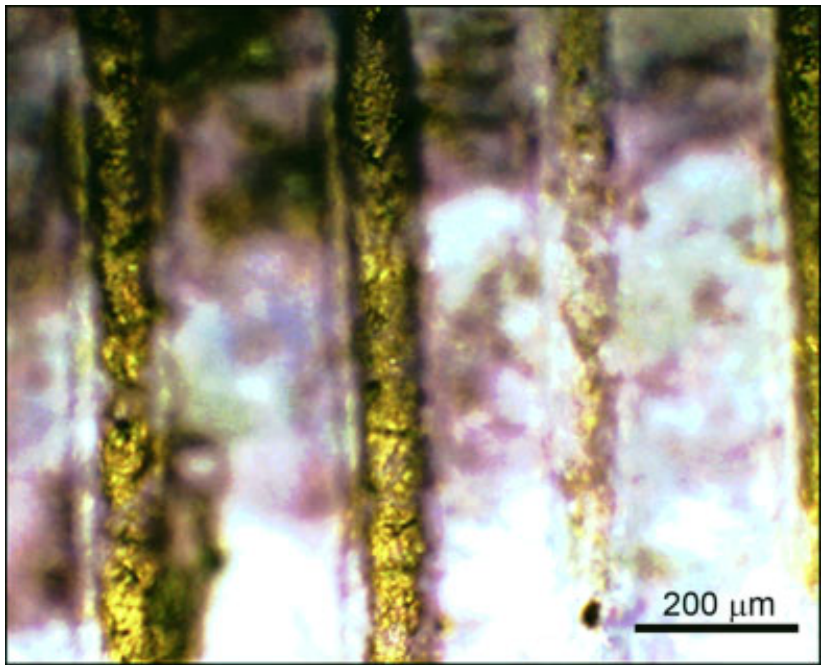

Figure 3. Reflective light microscopy image of micro-patterned and heparin-printed SPCL-HA membrane after toluidine blue staining

can enter into the grooves and transfer heparin-chitosan solution onto the surfaces of the microchannels by $\mu \mathrm{CP}$ transfer. The efficiency of heparin modification over the microchannel surfaces was determined by staining the membranes with toluidine blue. As seen by light microscopy, the heparin coating was determined to be predominantly over the microchannel surfaces (Figure 3). However, it can be seen that a minority of regions along the channel path were not stained. It is possible that small parts of the channel surfaces may not to be wetted during $\mu \mathrm{CP}$ transfer of heparin solution. However, this problem can be overcome by repeating the $\mu \mathrm{CP}$ process and eventually the heparin can cover all parts of the path. It is important that the coating must stay on the microchannels and not leach out during cell culture experiments (and for in vivo applications). The presence of stains on the surface of patterns after repeated washing of membranes with distilled water showed the durability of heparin. The cationic chitosan, which is not soluble at neutral and physiological $\mathrm{pH}$, must hold the anionic heparin molecules by means of complex formation and electrostatic interaction.

\subsection{Cell behaviour analysis}

\subsubsection{Cell culture on SPLC-HA membranes}

The orientation and morphology of fibroblasts on heparinprinted or -unprinted channel-patterned samples were analysed by SEM. The fibroblast cells which were cultured over heparin-printed SPCL-HA membranes showed very distinct morphologies when compared to unprinted regions (ridges). Outside the periphery of the microchannels, where there was no heparin, the fibroblasts were detected to form an uniform layer with normal cell morphology and were aligned randomly (Figure 4A). Over the microchannels, however, the fibroblast cells were stretched and they were bridging across the channel grooves, forming arch-like structures, after 2 days. As seen from Figure 4B, the fibroblast cells were covering most of the channel grooves, with occasional circular gaps, after 3 days of culture. In addition, it appears from the photomicrographs that the fibroblasts were covering the heparin-printed channels from the top only, and the inner parts were unpopulated.

In the case of micro-patterned SPCL-HA membranes without heparin coating, the morphology of the fibroblasts was seen to be unaltered on microchannels and ridge surfaces. As seen from Figure 4C, the fibroblasts were forming smooth cell layers all over the surface of ridge and grooves without making bridge formations after 3 days of culture. The growth pattern detected for heparin-printed channels was not detected for patterned membranes without heparin prints. In addition, the smooth, unpatterned membranes, which were used as controls (not shown in figures) also showed smooth
A

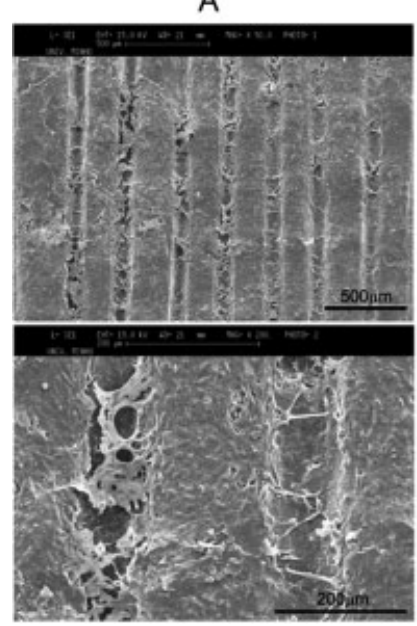

B

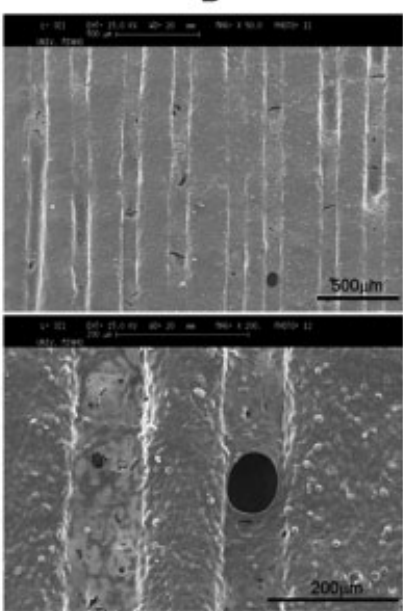

C

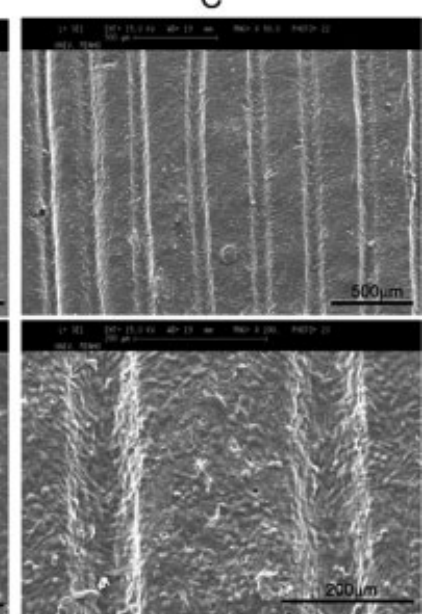

Figure 4. Fibroblast growth on heparin-printed channels of SPCL-HA membranes after 2 days (a), 3 days (b) and on micro-fabricated membranes without heparin print after 3 days of culture (c) 


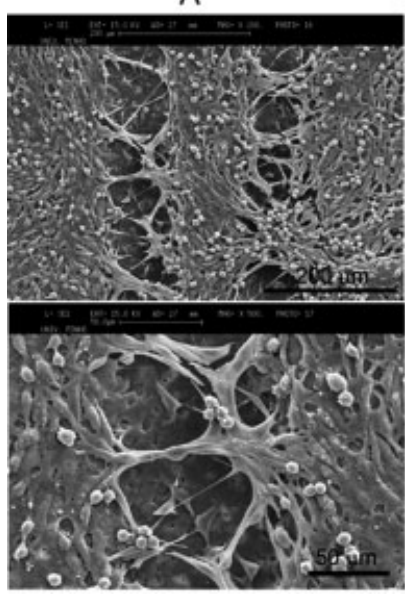

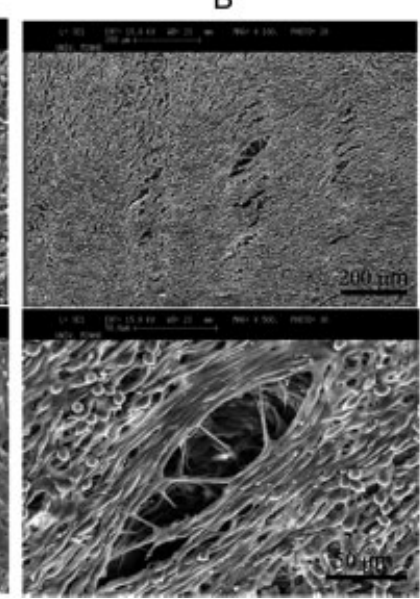

C

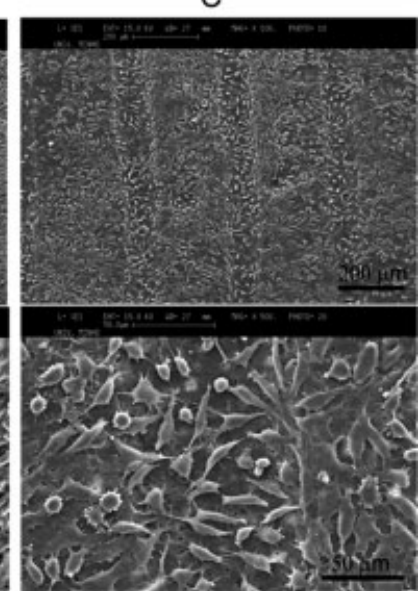

Figure 5. Fibroblast growth on micro-patterned and heparin-printed SPLA membranes after 3 days (a), 7 days (b) and on micro-fabricated membranes without heparin print after 3 days of culture (c)

cell morphology similar to that seen on micro-patterned membranes without heparin coating.

\subsubsection{Cell culture on SPLA membranes}

A similar influence of heparin imprinting on fibroblasts organization and morphology was observed on microchannel-patterned SPLA membranes as well. After 3 days of culture, the fibroblasts started bridging across the opposite edges of the heparin-printed channels by using their stretched filopodia (Figure 5A). After 7 days of culture, the fibroblasts were seen to cover the upper part the channels completely, even though the channel tracks could still be recognized, with occasional gaps (Figure 5B). Without heparin printing, however, there was no such kind of bridge formation or covering activity on top of the SPLA membrane channels, as seen after 3 days of cell culture (Figure 5C). As a result, it can be concluded that there was no effect of the variety of the biomaterials used on the cell response towards the two cues (microchannel patterning and heparin coating).

The cell behaviour analysis in this study showed that the biochemical cue (heparin) is a prerequisite for cells to recognize and act on the topographical cue (microchannel groove). Previously, the chemical environment rather than topographical factors was reported to be more important. For example, endothelial cells were detected to behave as they were on homogeneous sulphated hyaluronan substrate with topographical pattern, and only chemical differences among micro-domains induced a different and particular cell behaviour (Barbucci et al., 2002). In a similar study, 3T3 fibroblasts, bovine aortic and human endothelial cells orientated themselves more parallel to the chemical topography (Magnani et al., 2003). However, it was reported that the presence of laminin as a biochemical cue on grooves promoted neurite adhesion and outgrowth along the grooves in a synergistic way (Miller et al., 2002).

Our results suggest that fibroblasts can recognize a groove gap distance of a few cells size by forming cytoplasm extensions and cellular associations. It is possible that the biochemical cue initially triggered fibroblasts to recognize the size feature, which is greater than the average cell size $(10-30 \mu \mathrm{m})$ and facilitated them to cover a relatively long distances. Similar to our results, an unusual capability of ganglion neurons to extend across micropatterned grooves with feature sizes in the order of tens of microns has been shown (Goldner et al., 2006). It was reported that the highest bridge formation with neural cells was observed when providing a groove depth of $50 \mu \mathrm{m}$ and groove/plateau widths of 30 and $200 \mu \mathrm{m}$, respectively.

Interestingly, the fibroblasts covered the top of microchannels by forming tent-like structures without populating inside the channel where the heparin was coated. In general, the impaired cell spreading on heparin was reported and explained by the hydrophilicity and negative zeta potential, which in turn decreases protein adsorption and cell adhesion (Niepel et al., 2009). Also, heparin layers were shown to be rather cytophobic in the absence of adhesion-promoting proteins (Kirchhof et al., 2009). On the other hand, it was reported previously that in human skin heparin-chitosan complexes stimulate the wound-healing process (Kratz et al., 1997). In addition, the specific binding of fluorescent-labelled heparin to membrane $\mathrm{N}$-acetyl-glucosamine-6-sulphate receptor was demonstrated (Parisel et al., 2003). For these reasons, it is possible that the bridging facility of fibroblasts on heparin-printed hollow-channel structures may reflect a wound-healing process. These results, combined with the fact that naturally fibroblasts can interact with heparin at the cell membrane and be activated in the wound-healing process, suggest that both heparin and topographical cues may trigger fibroblasts to make organization on the channel structure.

In summary, our results showed that heparin printing on the channel surfaces triggered fibroblasts to recognize a topographical cue of several cell sizes and, consequently, promoted cellular organization in order to cover the gaps. It is apparent from SEM microphotographs that 
both topographical patterning and heparin printing are necessary for cells to cover the top of channels with a cell layer at late periods of cell culture.

\section{Conclusions}

Composite biomaterials membranes of SPCL with high hydroxyapatite content and SPLA were micro-patterned with parallel microchannels, using metal moulds and a hydraulic press. The microchannels produced had an average diameter of $102 \mu \mathrm{m}$ and interchannel spaces (ridges) of $174 \mu \mathrm{m}$. The heparin was spatially covered over the micro-patterns by $\mu \mathrm{CP}$ and subsequent toluidine blue staining indicated that the printing was predominantly over the microchannels. It was clearly observed that the heparin $\mu \mathrm{CP}$ printing triggered and activated fibroblasts and, in turn, the cells were organized spatially over the microchannels by bridging two sides of the grooves. In late cell culture periods, the fibroblasts completely covered the tops of the heparin-modified microchannels without cellular ingrowth. This study indicated that dual processing of composite biomaterials membranes with mechanical patterning and biochemical cue printing could be a potential tissue-engineering approach for controlled tissue development.

\section{Acknowledgements}

E. T. Baran thanks the Portuguese Foundation for Science and Technology (FCT) for providing a post-doctoral scholarship (No. SFRH/BPD/30768/2006). This work was partially supported by the FCT through funds from the POCTI and/or FEDER programmes and also by the European Union-funded STREP Project HIPPOCRATES (Grant No. NMP3-CT-2003-505758).

\section{References}

Adams JC, Schwartz MA. 2000; Stimulation of fascin spikes by thrombospondin- 1 is mediated by the GTPases, Rac and Cdc42. $J$ Cell Biol 150: 807-822.

Barbucci R, Lamponi S, Magnani A, et al. 2002; Micro-patterned surfaces for the control of endothelial cell behavior. Biomol Eng 19: 161-170.

Berry C, Campbell G, Spadiccino A, et al. 2004; The influence of microscale topography on fibroblast attachment and mobility. Biomaterials 25: 5781-5788.

Cao Y, Poon YF, Feng J, et al. 2010; Regulating orientation and phenotype of primary vascular smooth muscle cells by biodegradable films patterned with arrays of microchannels and discontinuous microwalls. Biomaterials 31: 6228-6238.

Csucs G, Michel R, Lussi JW, et al. 2003 Microcontact printing of novel copolymers in combination with proteins for cell-biological applications. Biomaterials 24: 1713-1720.

Flemming RG, Murphy CJ, Abrams GA, et al. 1999; Effects of micro- and nanostructured surfaces on cell behavior. Biomaterials 20: 573-588.

Ghibaudo M, Trichet L, Digabel JL, et al. 2009; Substrate topography induces a crossover from $2 \mathrm{D}$ to $3 \mathrm{D}$ behavior in fibroblast migration. Biophys $J$ 97: 357-368.

Goldner JS, Bruder JM, Li G, et al. 2006; Neurite bridging across micropatterned grooves. Biomaterials 27: 460-472.

Hasenbein ME, Andersen TT, Bizios R. 2002; Micro-patterned surfaces modified with select peptides promote exclusive interactions with osteoblasts. Biomaterials 23: 3937-3942.

Hollister SJ, Maddox RD, Taboas JM. 2002; Optimal design and fabrication of scaffolds to mimic tissue properties and satisfy biological constraints. Biomaterials 23(20): 4095-4103.

Kane RS, Takayama S, Ostuni E, et al. 1999; Patterning proteins and cells using soft lithography. Biomaterials 20: 2363-2376.
Kirchhof K, Hristova K, Krasteva N, et al. 2009; Multilayer coatings on biomaterials for control of MG-63 osteoblast adhesion and growth. J Mater Sci Mater Med 20: 897-907.

Kratz G, Arnander C, Swedenborg J, et al. 1997; Heparin-chitosan complexes stimulate wound healing in human skin. Scand $J$ Plast Reconstr Surg Hand Surg 31: 119-123.

Lauer L, Klein C, Offenhäusser A. 2001; Spot compliant neuronal networks by structure optimized micro-contact printing. Biomaterials 22: 1925-1932.

Lauffenburger DA, Horwitz AF. 1996; Cell migration: a physically integrated molecular process. Cell 84: 359-369.

Magnani A, Priamo A, Pasqui D, et al. 2003; Cell behavior on chemically microstructured surfaces. Mater Sci Eng C 23: 315-328.

Mahoney MJ, Chen RR, Tan J, et al. 2005; The influence of microchannels on neurite growth and architecture. Biomaterials 26: 771-778.

Manwaring ME, Walsh JF, Tresco PA. 2004 Contact guidance induced organization of extracellular matrix. Biomaterials 25: 3631-3638.

Miller C, Shanks H, Witt A, et al. 2001; Oriented Schwann cell growth on micro-patterned biodegradable polymer substrates. Biomaterials 22: 1263-1269.

Miller C, Jeftija S, Mallapragada S. 2002; Synergistic effects of physical and chemical guidance cues on neurite alignment and outgrowth on biodegradable polymer substrates. Tissue Eng 8: 367-378.

Niepel MS, Peschel D, Sisquella X, et al. 2009; pH-dependent modulation of fibroblast adhesion on multilayers composed of poly(ethylene imine) and heparin. Biomaterials 30: 4939-4947.

Nobes CD, Hall A. 1999; Rho GTPases control polarity, protrusion, and cell adhesion during cell movement. $J$ Cell Biol 144: $1235-1244$.
O'Brien FJ, Harley BA, Yannas IV, et al. 2004; Influence of freezing rate on pore structure in freeze-dried collagen-GAG scaffolds. Biomaterials 25: 1077-1086.

Oh SH, Kang SG, Kim ES, et al. 2003; Fabrication and characterization of hydrophilic poly(lactic-co-glycolic acid)/poly(vinyl alcohol) blend cell scaffolds by meltmolding particulate-leaching method. Biomaterials 24: 4011-4021.

Ostuni E, Yan L, Whitesides GM. 1999; The interaction of proteins and cells with selfassembled monolayers of alkanethiolates on gold and silver. Colloid Surfaces B 15: 3-30.

Papenburg BJ, Liu J, Higuera GA, et al. 2009; Development and analysis of multilayer scaffolds for tissue engineering. Biomaterials 30: 6228-6239.

Parisel C, Saffar L, Gattegno L, et al. 2003; Interactions of heparin with human skin cells: binding, location, and transdermal penetration. J Biomed Mater Res 67A: 517-523.

Parker KK, Brock AL, Brangwynne C, et al. 2002; Directional control of lamellipodia extension by constraining cell shape and orienting cell tractional forces. FASEB $J$ 16: 1195-1204.

Reignier J, Huneault MA. 2006; Preparation of interconnected poly( $\varepsilon$-caprolactone) porous scaffolds by a combination of polymer and salt particulate leaching. Polymer 47: 4703-4717.

Santos MI, Tuzlakoğlu K, Fuchs S, et al. 2008; Endothelial cell colonization and angiogenic potential of combined nanoand micro-fibrous scaffolds for bone tissue engineering. Biomaterials 29: 4306-4312.

Silva SS, Goodfellow BJ, Benesch J, et al. 2007; Morphology and miscibility of chitosan/soy protein blended membranes. Carbohyd Polym 70: 25-31.

Tan JL, Liu W, Nelson CM, et al. 2004; Simple approach to micropattern cells on common culture substrates by tuning substrate wettability. Tissue Eng 10: $865-872$. 
Thakar RG, Ho F, Huang NF, et al. 2003; Regulation of vascular smooth muscle cells by micro-patterning. Biochem Biophys Res Commun 307: 883-890.

Tuzlakoğlu K, Bolgen N, Salgado AJ, et al. 2005; Nano- and micro-fiber combined scaffolds: a new architecture for bone tissue engineering. J Mater Sci Mater Med 1: 1099-1104.

Zeltinger J, Sherwood JK, Graham DA, et al 2001; Effect of pore size and void fraction on cellular adhesion, proliferation, and matrix deposition. Tissue Eng 7(5): 557-572.
Zmora S, Glicklis R, Cohen S. 2002; Tailoring the pore architecture in 3D alginate scaffolds by controlling the freezing regime during fabrication. Biomaterials 23(20): 4087-4094. 